Artículo de investigación

\title{
Efectos de la sostenibilidad y del valor logístico en las relaciones entre empresas de transporte marítimo
}

Milva Eileen Justavino-Castillo

Profesora, Facultad de Ingeniería Industrial, Universidad Tecnológica de Panamá, David, Panamá. milva.justavino@utp.ac.pa

Irene Gil-Saura*

Catedrática, Facultad de Economía, Universidad de Valencia, Valencia, España. irene.gil@uv.es

María Fuentes-Blasco

Profesora Titular, Facultad de Ciencias Empresariales, Universidad Pablo de Olavide, Sevilla, España.

mfuebla@upo.es

\section{Resumen}

El objetivo de este trabajo fue analizar cómo la sostenibilidad (económica, social y ambiental), bajo el enfoque de triple línea base, afecta la calidad de servicio, el valor logístico y la satisfacción en empresas del sector marítimo. Se propone un modelo teórico que se contrasta con la información suministrada por una muestra de 50 responsables de empresas (transitarios, importadores y exportadores) en Panamá. La información se analiza mediante la estimación de regresión por mínimos cuadrados parciales, que permite confirmar el carácter multidimensional de la sostenibilidad y del valor logístico. Los resultados también confirman que las percepciones de los clientes sobre las iniciativas de sus proveedores, con base en este enfoque, poseen la capacidad de impulsar su satisfacción a través de la calidad y el valor; esto demuestra los beneficios de llevar a cabo tales iniciativas y, con ello, su alcance a nivel global.

Palabras clave: prácticas sostenibles; calidad de servicio; valor logístico; industria marítima; relaciones business to business.

\section{Effects of sustainability and logistic value in the relationship between ocean shipping companies}

\section{Abstract}

The aim of this study was to analyze how sustainability (economic, social, and environmental), based on the triple bottom line framework, influences on service quality, logistic value, and satisfaction between companies in the maritime sector. A theoretical model that is contrasted with the information provided by 50 company managers (freight forwarders, importers, and exporters) from Panama was proposed. The data is analyzed using partial least squares regression, which confirms the multidimensionality of sustainability and logistic value. The results also verify that the perceptions of customers about initiatives of their suppliers, based on this framework, can lead to an increase in customer satisfaction through service quality and value. The findings demonstrate the advantages to carry out such initiatives, and thus their global reach.

Keywords: sustainable practices; quality of service; logistic value; shipping industry; business-to-business relationships.

\section{Efeitos da sustentabilidade e valor logístico nas relações entre empresas de transporte marítimo}

\section{Resumo}

O objetivo deste trabalho foi analisar como a sustentabilidade (econômica, social e ambiental), sob a abordagem da tripla linha de base, afeta a qualidade do serviço, o valor logístico e a satisfação nas empresas do setor marítimo. Propõe-se um modelo teórico que contrasta-se com as informações fornecidas por uma amostra de 50 gerentes de empresas (transitários, importadores e exportadores) no Panamá. A informação é ánalisada por meio de estimação em regressão de mínimos quadrados parciais, o que permite confirmar a natureza multidimensional da sustentabilidade e do valor logístico. Os resultados também confirmam que as percepções dos clientes sobre as iniciativas dos seus fornecedores, com base nesta abordagem, têm a capacidade de conduzir a sua satisfação pela qualidade e valor; isso demonstra os benefícios de realizar tais iniciativas e, com isso, seu alcance global.

Palavras-chave: práticas sustentáveis; qualidade de serviço; valor logístico; indústria marítima; relações business to business.

* Autor para dirigir correspondencia

Clasificación: JEL: M31; M39; Q56.

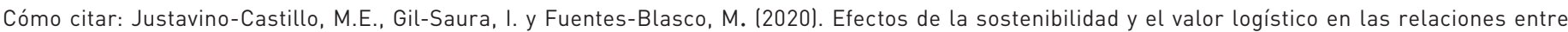
empresas de transporte marítimo. Estudios Gerenciales, 36(157), 377-390 https://doi.org/10.18046/j.estger.2020.157.3767

DOI: https://doi.org/10.18046/j.estger.2020.157.3767 


\section{Introducción}

Hasta finales del pasado siglo $X X$, el transporte de mercancías era visto como una actividad solo operativa; sin embargo, en la actualidad, la globalización de las actividades de producción y distribución genera un aumento en el volumen del comercio internacional que tiene como consecuencia la necesidad de acometer la gestión del transporte mediante su diferenciación estratégica, a través del cumplimiento de los tiempos de entrega y la reducción de los costos logísticos. Así, el transporte de mercancías se ha convertido en un facilitador clave de estas transacciones comerciales (Yuen, Wang, Wong y Zhou, 2017).

Entre los distintos modos de transporte, el marítimo es considerado el más eficiente, y es responsable de transportar aproximadamente el $80 \%$ del comercio mundial (Conferencia de las Naciones Unidas sobre Comercio y Desarrollo [UNCTAD], 2018). Aunque el transporte marítimo es el menos contaminante, ejerce una influencia significativa en el impacto medioambiental (Luttenberger y Luttenberger, 2017), que puede dañar de manera sustancial el ecosistema debido a su gran escala industrial (Lun, Lai, Wong y Cheng, 2015) y a la inmediatez del perjuicio que puede causar, por ejemplo, si llegase a ocurrir un accidente de barco que provoque el derrame del petróleo (Lam y Lai, 2015).

La Organización Marítima Internacional (OMI) estima que la emisión de dióxido de carbono causada por el sector marítimo internacional aumentará entre un 50\% y un $250 \%$ antes de 2050, aumento que dependerá del crecimiento económico (UNCTAD, 2016), y ha impuesto un plan para controlar el $\mathrm{CO}_{2}$ y otras sustancias nocivas emitidas por los buques. Además, en cuanto a la demanda, los clientes de servicios de envíos marítimos también comienzan a exigir a sus proveedores un mayor compromiso en torno al logro de sostenibilidad ambiental (Lam y Lai, 2015).

El envío sostenible implica satisfacer las necesidades del presente sin comprometer la capacidad de las generaciones futuras para cumplir sus propias necesidades, y requiere que las compañías navieras alcancen un equilibrio entre su desempeño económico, social y ambiental (Cheng, Farahani, Lai y Sarkis, 2015), para lograr una logística marítima sostenible (Psaraftis, 2016). Con este objetivo, muchas empresas han optado por asociarse en la búsqueda de soluciones conjuntas, por ejemplo, navieras como Hapag-Lloyd, American President Lines (APL), Maersk, y los cargadores como Home Depot, Mattel o Hewlett Packard (HP), forman parte del Grupo de Trabajo de Carga Limpia. Estas colaboraciones buscan conectar diferentes partes interesadas (fabricantes, intermediarios comerciales o transportistas de carga), para abordar los problemas de incumplimiento en los códigos de conducta y las regulaciones, a fin de mejorar el bienestar en la comunidad (Lun, Lai, Wong y Cheng, 2016). Estas iniciativas ponen de manifiesto que, para conseguir resultados óptimos en la gestión de prácticas sostenibles, es importante la colaboración entre empresas. Además, existen evidencias de que la aplicación de prácticas sostenibles puede mejorar la retención de los clientes y, con ello, alcanzar un mejor desempeño económico (Shin, Thai, Grewal y Kim, 2017). En este contexto, conocer el efecto que produce implementar prácticas sostenibles es de especial importancia, sobre todo en países en los que el transporte marítimo representa una parte sustancial de su economía, como es el caso de Panamá.

Desde 1914, con la construcción del Canal de Panamá, este país ha contribuido al dinamismo del comercio internacional y se ha convertido en una pieza importante a nivel mundial, al recortar distancias y tiempos de comunicación en el transporte marítimo, gracias a su privilegiada posición geográfica.

En esta dirección, este trabajo persigue analizar los beneficios de la aplicación de prácticas sostenibles, desde su triple perspectiva: económica, social y medioambiental, y determinar cómo estas prácticas influyen en la calidad de servicio, el valor logístico y la satisfacción; todo ello observado desde un enfoque de relaciones entre empresas (business to business o B2B). Se toma como punto de partida las percepciones de las empresas clientes, a partir de la opinión de responsables de empresas importadoras, exportadoras y transitarias en la República de Panamá.

En relación con la metodología, para el desarrollo de este trabajo se realizó una investigación cuantitativa, seguida de los correspondientes análisis que permitieron, en combinación con un análisis factorial exploratorio (AFE) y un análisis factorial confirmatorio (AFC), validar las escalas $y$, finalmente, probar el modelo de medida que se propone, a través del método de regresión de mínimos cuadrados parciales.

El trabajo se estructura como sigue: en primer lugar, se presenta el marco teórico, en el que se delimitan los fundamentos de la investigación a partir de una breve revisión de la literatura sobre los constructos de interés, esto es, prácticas sostenibles, calidad de servicio, valor logístico y satisfacción; además, se plantea un modelo teórico que identifica relaciones en cadena entre las variables objeto de estudio. Segundo, se describe la metodología de investigación, la cual se fundamenta en una encuesta a partir de las opiniones de las empresas clientes, y se define el contexto de la investigación empírica. Tercero, se presentan los resultados, que combinan análisis exploratorios y confirmatorios, junto con la estimación de las relaciones causales entre los principales constructos. Por último, se exponen las conclusiones y se reflexiona en torno a las oportunidades de investigación derivadas.

\section{Marco teórico}

En esta sección, tal y como ha sido anunciado, se procede a revisar los fundamentos teóricos de la investigación. Para ello, en primer lugar, se delimitan las funciones de los distintos agentes que intervienen en el transporte marítimo, para ello, se establecen los roles de empresa proveedora y cliente analizados en este trabajo. A continuación, se revisan los tópicos en los que se apoya la secuencia de relaciones que sustenta el modelo teórico, esto es, las prácticas sostenibles desde el enfoque de 
triple línea base (TBL, por sus siglas en inglés), la calidad de servicio, el valor logístico y la satisfacción, todo ello en el contexto de las relaciones entre empresas B2B.

\subsection{Agentes que intervienen en el transporte marítimo}

En el sistema logístico marítimo se producen vínculos entre las empresas proveedores y las empresas clientes. Dentro de este sistema, se incluyen como agentes activos los transitarios (freight forwarder). Estos agentes asumen, entre otras, funciones tales como la gestión del transporte, los seguros y la tramitación de la documentación asociada o el diseño de las rutas de envío. Por otro lado, las navieras se encargan de todos los trámites que tengan que cumplir los buques en puerto, por ejemplo, tareas comerciales, solicitudes de atraque, relaciones con autoridades portuarias, etc. En este contexto, actúan igualmente las empresas importadoras y exportadoras, que contratan los servicios prestados tanto por los transitarios como por las navieras; estas últimas son contratadas, ya sea directa o indirectamente, a través del transitario, con el objeto de transportar sus mercancías (Cabrera-Cánovas, 2011). Así, esta investigación reconoce como expedidores a todos los potenciales clientes de las compañías navieras: los exportadores, importadores y transitarios, con el fin de estudiar las creencias y actitudes que tienen los expedidores sobre el nivel de servicio que les ofrece su principal compañía naviera, en cuanto proveedora.

\subsection{Prácticas sostenibles en el transporte marítimo}

Cada vez es más habitual que las empresas incorporen en sus operaciones la sostenibilidad (Ahi y Searcy, 2015). Las presiones sociales, competitivas y las distintas legislaciones aumentan el interés y la necesidad de las empresas en prestar una mayor atención a los efectos medioambientales y sociales de sus actividades en la cadena de suministro (Stindt, 2017). Incluso muchas navieras han adoptado prácticas ecológicas de envío (GSPs-Green Shipping Practices). Las GSP incluyen el cálculo de la huella de carbono de las rutas de envío y el uso de equipos de envío alternativos con el objetivo de reducir el daño ambiental en el transporte de cargas (Lun et al., 2016).

Por otra parte, la gestión de una cadena de suministro sostenible (SSCM) es definida como la gestión de sostenibilidad que busca crear valor económico en la cadena de suministro con bajo impacto negativo social y ambiental (Schaltegger y Burritt, 2014). Kleindorfer, Singhal y Van Wassenhove (2005) describieron la SSCM como "la integración de las ganancias, las personas y el planeta en la cultura, la estrategia y las operaciones de las empresas" (p. 482). Algunos autores consideran que implementar la sostenibilidad requiere de un enfoque de $T B L$, con el que se buscan mejoras del desempeño en las dimensiones medioambientales, económicas y sociales (Ahi y Searcy, 2015), por lo que se establece que a partir de la SSCM se deduce el TBL (Stimdt, 2017). Otros autores evalúan estas dimensiones de sostenibilidad desde las " $3 \mathrm{P}$ ", esto es, Planeta, Personas y Profit (ganancias) (Asif, Searcy, Ambika y Ahmad, 2011). Algunas aproximaciones se sitúan en la perspectiva Green SCM (GSCM) o Corporate Social Responsibility (CSR); sin embargo, las diferencias entre todas ellas no están suficientemente justificadas (Stindt, 2017). Al respecto, Gopalakrishnan, Yusuf, Musa, Abubakar y Ambursa (2012) mencionan que "ambos pueden verse como subconjuntos de SSCM. Mientras GSCM se encuentra en la interfaz entre la litosfera, la tecnosfera y la biosfera, la CSR se interpreta como la dimensión social del negocio sostenible" (citado en Stindt, 2017, p. 147). La adopción de prácticas sostenibles contribuye a la mejora de la eficiencia corporativa y proporciona un mayor nivel de confianza entre las partes interesadas (Mitchell, Wooliscroft y Higham, 2010). De esta forma, la CSR se convierte en un punto clave para mejorar estas relaciones, generar lealtad hacia el cliente y ganar ventajas competitivas.

Con todo, se observa que la sostenibilidad abarca distintas áreas y presenta diferentes denominaciones. A continuación, se detallan las actividades de gestión sostenible con base en el enfoque TBL y se distingue entre actividades económicas, sociales y ambientales.

\subsubsection{Actividades económicas}

La relación entre ser "verde" y ser una empresa económicamente sostenible ha sido un tema analizado por diversos autores a lo largo del tiempo (Synnestvedt y Schaltegger, 2002). Los términos crecimiento económico y desarrollo sostenible a menudo se usan indistintamente; sin embargo, existen diferencias entre ambos. El crecimiento económico se refiere a un cambio en el tamaño de la economía, mientras que el desarrollo sostenible, como parte del TBL, se define como programas, políticas o actividades diseñadas para crear o retener empleos y riqueza que contribuyan a limitar problemas ambientales, sociales y económicos a lo largo del tiempo (Hammer y Pivo, 2017). Montabon, Sroufe y Narasimhan (2007) consideran los beneficios del desempeño económico sostenible, pues, al aplicar un envío sostenible con economías de escala, el rendimiento global aumentaría debido a los ajustes en el costo, la energía y la eficiencia operativa; por otra parte, se obtendría un ahorro de costos debido a la reducción del consumo de energía y materiales. La gestión ambientalmente sostenible es un tema importante porque, por un lado, las empresas obtienen beneficios y cuota de mercado, lo que es en un determinante clave de la posición competitiva, y, por otro, se comprometen a proteger el medioambiente (Lun et al., 2015), esto evidencia la interrelación existente entre los tres enfoques de la TBL. Así, la gestión ambiental en el sector marítimo permite que las empresas mejoren sus posiciones competitivas, mientras logran reducir los efectos negativos de sus operaciones mediante la aplicación de las prácticas de envío verde.

\subsubsection{Actividades sociales}

La sostenibilidad social significa que las organizaciones agregan valor a sus comunidades al aumentar 
el capital humano y fomentar el capital social (Dyllick y Hockerts, 2002). Según Sarkis, Helms y Hervani (2010), la sostenibilidad social enfatiza la gestión de los recursos sociales, incluidas las habilidades de las personas, las instituciones, las relaciones y los valores sociales. Además, hay estudios que indican la existencia de una relación entre la sostenibilidad social y la CSR, aunque los términos se han usado con frecuencia indistintamente (Hutchins y Sutherland, 2008). Según Wan, Young, Kim, Ei y Kim (2016), la CSR son las prácticas comerciales que voluntariamente adoptan las empresas, puesto que tienen la responsabilidad ética de tratar al público y al medioambiente con dignidad y respeto. La CSR se ha analizado mediante cinco dimensiones: medioambiental, social, económica, stakeholders y de voluntariado (Yuen y Thai, 2017). Aunque las dimensiones de la CSR no difieren considerablemente entre las industrias (Lu, Lin y Tu, 2009), hay evidencia de que los factores ambientales sostenibles, por lo general, se abordan mediante una evaluación de impacto, pero los aspectos sociales rara vez se consideran (Sarkis et al., 2010). Incluso las empresas navieras ponen mayor énfasis en la gestión del medioambiente que en los problemas sociales (Pawlik, Gaffron y Drewes, 2012). Su justificación se basa en que las mejoras en el desempeño ambiental se asocian más estrechamente con las mejoras financieras (Yuen y Thai, 2017). Sin embargo, las compañías navieras, para satisfacer a sus clientes, se ven obligadas a integrar las preocupaciones sociales y ambientales en su estrategia comercial y operacional (Yuen y Thai, 2015a).

\subsubsection{Actividades ambientales}

El movimiento de las mercancías desde su producción hasta el consumo se conforma a partir de una secuencia de actividades que contribuyen al comercio mundial y ayudan al desarrollo económico de los países. Sin embargo, estas actividades relacionadas pueden causar daños ambientales (Lun et al., 2015). Para satisfacer la búsqueda del cliente por la sostenibilidad ambiental en las operaciones de envío, las empresas navieras deben comprender lo que los clientes (expedidores) esperan y requieren de ellos en las dimensiones ambientales y determinar cómo esos requisitos pueden traducirse en sus procesos (Lam y Lai, 2015).

El sector marítimo presenta grandes retos desde el punto de vista ambiental. En la actualidad, se produce un alto nivel de óxidos de azufre (SOx), óxidos de nitrógeno (NOx) y de materia particulada en el transporte de mercancías, sobre todo cuando no se tienen tecnologías apropiadas para su reducción. Entre tales óxidos, el NOx es el causante del esmog (mezcla de humo y polvo suspendidol y del calentamiento global. Por otra parte, las emisiones de ruido, el ruido subacuático y las colisiones con mamíferos marinos, los derrames de hidrocarburos, desechos y los materiales que son liberados durante el desguace de buques también son factores generadores del impacto ambiental producido por el transporte marítimo (Luttenberger y Luttenberger, 2017).
Por lo tanto, las causas de la contaminación ambiental no solo perjudican a los accionistas de la industria naviera, sino también a la industria pesquera y a la turística, lo que indica que la CSR está asociada con el concepto de calidad; así que los servicios de calidad del transporte marítimo deben incorporar esta dimensión. En este sentido, se enfatiza en que "la calidad de servicio en el transporte marítimo está estrechamente relacionada con la seguridad y la protección del medioambiente" (Thai, 2008, p. 501).

Yuen y Thai (2017) señalan que hay muchas similitudes entre calidad de servicio y CSR en términos de identificación, implementación y comunicación de las actividades de la cadena de valor que los clientes consideran importantes. La interacción que exista entre los atributos que forman parte de la industria de envío por transporte marítimo es la que define el proceso y los resultados (Yuen y Thai, 2015b); interacción que puede ser aditiva (sin interacción), compensatoria o sinérgica (Yuen y Thai, 2017). Así, la teoría de la contingencia busca lograr el ajuste entre elementos (variables) de una teoría, y una forma de definir este ajuste es a través del signo de la interacción entre el predictor y la variable de contingencia. Un buen ajuste lleva implícito un signo positivo o interacciones sinérgicas, mientras que un ajuste deficiente es determinado por un signo negativo o interacciones compensatorias. Según estudios realizados por Yuen y Thai (2017) para conocer el grado de ajuste entre las actividades de CSR y la calidad de servicio, los resultados muestran que la CSR complementa la calidad de servicio. Por otra parte, hay interacciones sinérgicas entre calidad de servicio y CSR. Por todo lo anterior, en el siguiente epígrafe se revisará esta variable de interés.

\subsection{Calidad de servicio}

Entender calidad de servicio es fundamental para comprender su aplicabilidad en el estudio del servicio logístico (Gil-Saura, Berenguer-Contri, Ruiz-Molina y Ospina-Pinzón, 2017; Gil-Saura, Berenguer-Contri y Ruiz-Molina, 2018). La calidad del servicio percibida se interpreta como las creencias generales de un cliente sobre el servicio recibido frente a la perspectiva de análisis centrada en la calidad objetiva. Esta última se observa en el cumplimento de estándares, especificaciones y procedimientos (Gil-Saura et al., 2017). Así, los estándares genéricos declarados por la Organización Internacional de Normalización (ISO), calidad y medioambiente (ISO 9001 e ISO 14000), deben ser aplicados por los socios de la cadena de suministro del sector marítimo (Celik, 2009). Las normas ISO 14000 e ISO 26000 (certificación de gestión social) son utilizadas como indicadores de desempeño de sostenibilidad por los expedidores y como criterios de selección de sus compañías navieras (Pawlik et al., 2012). Para Srdoč, Bratko y Sluga (2007), la aplicación de los estándares de calidad ISO en las empresas del sector marítimo es muy útil para mejorar la calidad del servicio y la satisfacción del cliente en el mercado.

En este trabajo se considera la calidad de servicio como subjetiva y, por tanto, se entiende como una evaluación que 
compara las expectativas del cliente y las percepciones que tiene sobre el desempeño del servicio (Yuen y Thai, 2015a; Gil-Saura et al., 2017).

Para el sector del transporte marítimo, la calidad de servicio puede verse como estrategia de diferenciación, mediante la entrega de alta calidad (Gil-Saura et al. 2017; Yuen y Thai, 2017). La calidad de envío de mercancías por transporte marítimo puede ser analizada desde dos dimensiones (Yuen y Thai, 2017): rendimiento del tiempo y experiencia del cliente. La dimensión del rendimiento del tiempo comprende confiabilidad del cronograma, frecuencia de navegación y velocidad (Notteboom, 2006). Por otra parte, la dimensión de experiencia del cliente se enfoca en obtener la identidad de marca y la lealtad (Shin y Thai, 2015; Yuen y Thai, 2017). A partir de recursos tanto tangibles como intangibles, es posible lograr los objetivos de esta dimensión.

Según la revisión de literatura realizada por Yuen y Thai (2017), los recursos tangibles que son atractivos para los transportistas incluyen la presencia de certificados ISO, condiciones físicas de oficinas y equipos, la apariencia de los empleados de primera línea, la proximidad de la oficina del transportista de contenedores y la disponibilidad de recursos de tecnología, por ejemplo los sitios web que facilitan la reserva, el pago y los reclamos (Kannan, Bose, y Kannan, 2011). Los recursos intangibles se refieren a los elementos no observables de un servicio de envío: empatía, receptividad y seguridad al interactuar con los clientes. Otras propuestas como la de Thai (2008) establecen un modelo conceptual de calidad de servicio en el transporte marítimo que consta de 6 dimensiones y 24 factores, que dan como resultado que la eficiencia en la gestión y la CSR son importantes para indicar la calidad del servicio de las organizaciones de transporte marítimo.

Muchos modelos se han propuesto para medir la calidad de servicio; sin embargo, el más destacado, puesto que da origen a la prolífica tradición de investigación de la escuela americana, es el de Parasuraman, Zeithaml y Berry (1985). Esta tradición de investigación tiene como base una escala de medición de calidad del servicio (SERVQUAL), que delimita el concepto a partir de cinco dimensiones: confiabilidad, capacidad de respuesta, seguridad, empatía y elementos tangibles.

La aplicación de la escala SERVQUAL para la medición de la calidad en el servicio de transporte presenta algunos inconvenientes (Chen, Chang, y Lai, 2009; Yuen y Thai, 2015b; Gil-Saura et al., 2017, 2018). Por una parte, los indicadores son genéricos, por lo que su enfoque no se centra en la industria del transporte marítimo, y, por otra, se define a partir de las actitudes mostradas por el consumidor final, a diferencia del modelo de servicio del transporte marítimo, en el que intervienen empresas y los informantes son empleados representantes de las distintas organizaciones clientes (Gounaris, 2005).

Con la finalidad de identificar las dimensiones de la calidad en el servicio de transporte regular marítimo, Yuen y Thai (2015a) decidieron validar los indicadores desarrollados por Sureshchandar, Rajendran y
Anantharaman (2002) para el transporte marítimo, a través de entrevistas realizadas a las compañías de transporte de contenedores y sus clientes, agentes de carga y fabricantes. Los resultados indicaron que la calidad de servicio y la responsabilidad social influyen de manera positiva, y son antecedentes de la satisfacción del cliente, lo que coincide con otros estu-dios realizados en el sector de envíos marítimos (Panayides, 2003; Lindstad, Asbjørnslett y Strømman 2016); aunque el principal contribuyente a la satisfacción del cliente en el envío sigue siendo la calidad del servicio. Además, otros estudios señalan que hay una relación positiva entre la calidad del servicio logístico y el valor logístico (ServeraFrancés, Gil-Saura y Fuentes-Blasco, 2008; Gil-Saura et al., 2018); a continuación se identifica el marco conceptual de esta última variable.

\subsection{Valor logístico}

Proporcionar valor a los clientes contribuye a maximizar la satisfacción y lealtad de los usuarios (Helgesen, 2006); de esta manera, se crea una ventaja competitiva sostenible para retener clientes (Schellinck y Brooks, 2016). En el ámbito de la logística, la introducción del concepto valor "es considerada como revolucionaria a la vez que fundamental, tanto para académicos como para profesionales" (Servera-Francés, Arteaga-Moreno y Gil-Saura, 2011, p. 95). El término valor es definido por Rutner y Langley (2000) como un concepto abstracto, es decir, intangible, y cuya definición variará de acuerdo con el contexto y percepción de los administradores. Según Servera-Francés et al. (2011), el análisis del valor logístico debe realizarse desde el enfoque del valor percibido, y desde una orientación de juicio evaluativo, haciendo hincapié en que el valor reside en la experiencia del uso o consumo (Woodruff, 1997). El valor logístico se consigue cuando las expectativas y requisitos de los clientes relacionados con el servicio logístico se satisfacen al menor costo posible (Novack, Langley y Rinehart, 1995; Rutner y Langley, 2000; Gil-Saura et al., 2018).

En el sector marítimo, el valor logístico se logra cuando el sistema cumple con las necesidades del cliente. El sistema lo constituyen entonces las empresas proveedoras de servicios, quienes deben identificar las demandas y necesidades de cada uno de sus clientes. En este sector, existen distintos perfiles de clientes, todos ellos constituyen la cadena de suministro. Así, por ejemplo, se identifican los cargadores, quienes demandan los servicios de carga; los transitarios, que trabajan para los cargadores y a la vez son clientes de las navieras; y las líneas navieras, que son los clientes de los operadores de puertos y terminales.

El rendimiento de los servicios logísticos marítimos dependerá del comportamiento de todo el sistema y su éxito o fracaso repercutirá en todo el proceso, ya que los elementos que lo constituyen están conectados entre sí. Por lo tanto, a mayor valor logístico marítimo, mayor satisfacción del cliente y del sistema (Eon-Seong y Dong-Wook, 2010). 


\subsection{Satisfacción del cliente}

Según Oliver (1980), la satisfacción del cliente es una reacción cognitiva y afectiva a un incidente del servicio. Comparar la experiencia de un cliente con su expectativa en un encuentro de servicio se considera una respuesta de cumplimiento (Yuen y Thai, 2015a). Otros autores consideran la satisfacción como una unidad de medida basada en la comparación entre la percepción de los resultados y ciertos estándares de comparación, ya que esto va a cambiar dependiendo del estudio que se realice. Las variables comparativas utilizadas han sido las expectativas, los deseos, las necesidades y las basadas en la experiencia. Según la literatura, las expectativas del cliente son la variable más utilizada (Gil-Saura et al., 2018).

En el transporte marítimo, la satisfacción del cliente se deriva de la realización de actividades de apoyo, que, por lo general, se brindan antes y después de un servicio de transporte. Algunas actividades de apoyo podrían ser la satisfacción previa a un servicio, la satisfacción que ocurre después de un servicio de transporte, la facilidad y rapidez de las reclamaciones, en caso de pérdidas de mercancías o entregas tardías (Yuen y Thai, 2015a). Satisfacer a los clientes en el transporte regular marítimo podría resultar complejo por la cantidad de actores que intervienen al momento de seleccionar un servicio marítimo (Frankel, 1993). Por ejemplo, la selección de las rutas y la combinación de tipos de transporte lo realiza el transitario en nombre del exportador o importador. Por tanto, aunque el exportador o importador es, en el contexto de este trabajo, el cliente final, también es importante que las empresas navieras satisfagan las necesidades de calidad de sus clientes inmediatos: los transitarios (Yuen y Thai, 2015a).

En síntesis, y a partir de todo lo expuesto, la revisión de la literatura ha mostrado evidencias de que las prácticas sostenibles, analizadas desde sus tres dimensiones (social, económica y ambiental), tienen un impacto positivo en la generación de calidad de servicio en el transporte marítimo (Thai, 2008; Yuen y Thai, 2015a, 2017). También, distintas investigaciones han identificado la calidad de servicio como antecedente directo del valor logístico y la satisfacción como la consecuencia inmediata (Servera-Francés et al., 2008; Gil-Saura et al., 2018). De esta forma, es posible proponer las siguientes hipótesis de investigación:

- H1: las prácticas sostenibles inciden directa y positivamente sobre la calidad de servicio.

- H2: la calidad del servicio logístico incide directa y positivamente sobre el valor logístico.

- H3: el valor logístico incide directa y positivamente sobre la satisfacción del cliente.
En la figura 1 se presenta el modelo que propone las relaciones planteadas en forma de hipótesis.

\section{Metodología}

En este apartado se define el contexto en el que se desarrolla la investigación. Primero, se presenta una descripción de la población a la que se dirigió, luego, se explica el proceso de recolección de información y se presentan las escalas utilizadas para medir los constructos, para lo cual se identificaron las dimensiones que las definen, así como los ítems empleados en el cuestionario. Para finalizar, se exponen las técnicas estadísticas empleadas en el análisis de la información, destacando la estimación de las relaciones de causalidad recogidas en la cadena de efectos de la figura 1. Entre otras razones, la elección de esta metodología radica en el uso de escalas de medida para construir variables latentes y estimar la relación directa e indirecta entre ellas.

\subsection{Muestra y recogida de información}

Con la finalidad de analizar la percepción que tienen los expedidores de la sostenibilidad que les ofrece su principal compañía naviera, y confirmar las hipótesis del modelo propuesto, en términos de efectos sobre el resto de las variables bajo observación, se llevó a cabo un estudio cuantitativo basado en un cuestionario estructurado. La recolección de información se realizó en la República de Panamá mediante encuesta personal dirigida a 50 encargados de gestionar el transporte de sus mercancías a través de la naviera de su preferencia.

La recolección de los datos se llevó a cabo entre los meses de enero y marzo del 2019. Un 68\% de las empresas investigadas son transitarios, mientras que en un $18 \%$ de los casos su negocio se centra en las importaciones y solo el $4 \%$ se dedica a las exportaciones. La mayoría de las empresas encuestadas (32\%) tiene entre 6 y 10 años de experiencia en el sector del transporte marítimo y solo dos empresas encuestadas tienen más de 500 empleados. En la tabla 1 se muestran las principales características de la investigación. Como paso previo a la recopilación definitiva de la información, se realizó una preprueba a diez empresas, en las que los cuestionarios fueron respondidos por profesionales del sector logístico. El análisis de estos resultados permitió mejorar el enunciado de algunos indicadores en el cuestionario.

Con base en la información recogida, se realiza un AFE, un AFC y por último se estiman los parámetros de un modelo de ecuaciones estructurales, con el fin de contrastar las hipótesis planteadas.

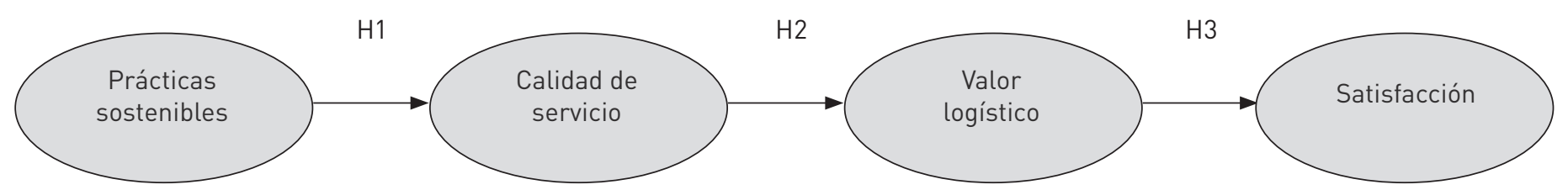

Figura 1. Modelo teórico e hipótesis

Fuente: elaboración propia. 
Tabla 1. Principales características de la investigación

\begin{tabular}{|c|c|c|c|}
\hline $\begin{array}{l}\text { Universo } \\
\text { Ámbito geográfico }\end{array}$ & \multicolumn{3}{|c|}{$\begin{array}{l}\text { Responsables de las compras y envíos en el sector marítimo, } \\
\text { expedidores. }\end{array}$} \\
\hline Tamaño muestral & \multicolumn{3}{|l|}{ República de Panamá } \\
\hline Diseño muestral & \multicolumn{3}{|l|}{50 empresas } \\
\hline Periodo de recogida de datos & \multicolumn{3}{|l|}{ Entrevista personal } \\
\hline Técnicas estadísticas & \multicolumn{3}{|l|}{ Enero-marzo 2019} \\
\hline Programas estadísticos & \multicolumn{3}{|l|}{ Análisis descriptivo } \\
\hline & \multicolumn{3}{|c|}{ Análisis Factorial Exploratorio y Confirmatorio (AFE, AFC) } \\
\hline & \multicolumn{3}{|c|}{ Modelo de Ecuaciones Estructurales } \\
\hline & \multicolumn{3}{|c|}{ IBM SPSS Statistics 22; Smart PLS 3.0} \\
\hline Perfil de las empresas & & Número & $\%$ \\
\hline \multirow[t]{4}{*}{ Tipo de negocio } & Exportador & 2 & 4 \\
\hline & Importador & 9 & 18 \\
\hline & Transitarios & 34 & 68 \\
\hline & Otros & 5 & 10 \\
\hline \multirow[t]{6}{*}{ Años de servicio de la empresa } & $1-5$ & 11 & 22 \\
\hline & $6-10$ & 16 & 32 \\
\hline & $11-15$ & 5 & 10 \\
\hline & $16-20$ & 2 & 4 \\
\hline & Más de 20 años & 10 & 20 \\
\hline & Empresas que no respondieron & 6 & 12 \\
\hline \multirow[t]{6}{*}{ Número de colaboradores } & $1-25$ & 24 & 48 \\
\hline & $26-50$ & 13 & 26 \\
\hline & $51-100$ & 6 & 12 \\
\hline & $101-250$ & 5 & 10 \\
\hline & $501-1000$ & 1 & 2 \\
\hline & Más de 1000 & 1 & 2 \\
\hline
\end{tabular}

Fuente: elaboración propia.

\subsection{Medición de las variables}

Todas las variables incluidas en el estudio han sido medidas mediante ítems ya validados en la literatura y adaptados a esta investigación (tabla 2). Se emplearon escalas tipo Likert de 7 puntos (siendo 1 "totalmente en desacuerdo" hasta 7 "totalmente de acuerdo") en las que el encuestado debía posicionarse según su grado de conformidad con las afirmaciones propuestas.

Tabla 2. Escalas de medida de las variables en el cuestionario

\begin{tabular}{lll}
\hline Variable & $N^{0}$ de ítems & Escala \\
\hline Prácticas sostenibles & 16 & Shin et al. (2017) \\
Calidad de servicio & 4 & Yuen y Thai (2017) \\
Valor logístico & 13 & Servera et al. (2008) \\
Satisfacción & 4 & Shin et al. (2017) \\
\hline
\end{tabular}

Fuente: elaboración propia.

Los ítems relativos a las prácticas sostenibles han sido extraídos del trabajo de Shin et al. (2017) y se contemplan las actividades económicas (cinco ítems), sociales (cinco ítems) y medioambientales (seis ítems); por lo tanto, para la medición de las prácticas sostenibles se utilizaron 16 ítems. La calidad del servicio fue medida por cuatro ítems extraídos de la propuesta de Yuen y Thai (2017). En cuanto a la escala utilizada para medir el valor logístico, se aplicó la escala utilizada por ServeraFrancés et al. (2008), que evalúa el concepto a través de 13 ítems. Por último, la satisfacción se midió mediante cuatro ítems extraídos de la investigación de Shin et al. (2017) (anexos, tabla A1). Además, se han incluido dos variables de control en el modelo, el tipo de negocio de la empresa y la antigüedad de la empresa.

\subsection{Análisis estadístico de la información}

Para el análisis de la información se emplean diferentes técnicas estadísticas destinadas a comprobar la validez de las escalas y probar las hipótesis propuestas. Concretamente, la dimensionalidad de las escalas se estudia mediante un AFE con rotación Varimax y utilizando los criterios de valores propios mayores que uno (Hair, Anderson, Tatham y Black, 2008). Esta técnica permite comprobar si los ítems de medida cargan a su dimensión correspondiente. Luego, se aplicó un AFC que permite confirmar la dimensionalidad y comprobar las propiedades psicométricas de las escalas.

Tras verificar la fiabilidad y validez de las escalas de medida, se procede a verificar las hipótesis planteadas mediante la estimación de un modelo de ecuaciones estructurales. Este tipo de modelización permite considerar en conjunto la medición de los constructos y estimar las relaciones causales o efectos entre las variables latentes, sin contaminación por errores de medición (BatistaFoguet y Coenders-Gallart, 2000). Presentada en forma de técnica avanzada de otros análisis multivariantes, como la regresión lineal múltiple y el análisis factorial, permite representar las relaciones entre variables no observables 
o latentes (prácticas sostenibles, valor logístico, calidad de servicio y satisfacción) y estimar relaciones de dependencia múltiple (Hair et al., 2008).

\section{Análisis de resultados}

4.1 Análisis preliminar de la dimensionalidad de las escalas de medida

Para analizar la dimensionalidad de las escalas se procedió en distintas fases. En primer lugar, se realizó un AFE de componentes principales por escala, utilizando el software IBM SPSS 22.0, con el objetivo de estudiar la dimensionalidad preliminar y, si fuera conveniente, depurar los ítems que así lo requieran por falta de carga a su factor.

De esta forma, se comprueba que los ítems utilizados para medir el constructo sostenibilidad se agrupan en tres factores que, atendiendo su contenido, se han denominado dimensión de actividades económicas, sociales y medioambientales, que explican en conjunto el $69,47 \%$ de la variabilidad total (KMO=0,880; prueba de esfericidad de Barlett: Chi-cuadrado=555,37 [gl=136], p-valor $<0,0001$ ]. Por tanto, atendiendo a la propuesta inicial de Shin et al. (2017), se retienen los 16 ítems adaptados, como se observa en la figura 2.

Replicando el análisis exploratorio en la escala destinada a medir el valor logístico, los resultados indican que se deben retener 13 ítems de los 14 planteados en la escala original y se extraen tres factores. Siguiendo a ServeraFrancés et al. (2008), se han denominado productividad del servicio logístico, importancia del servicio logístico y cuantificación del valor logístico (figura 3). Conjuntamente,

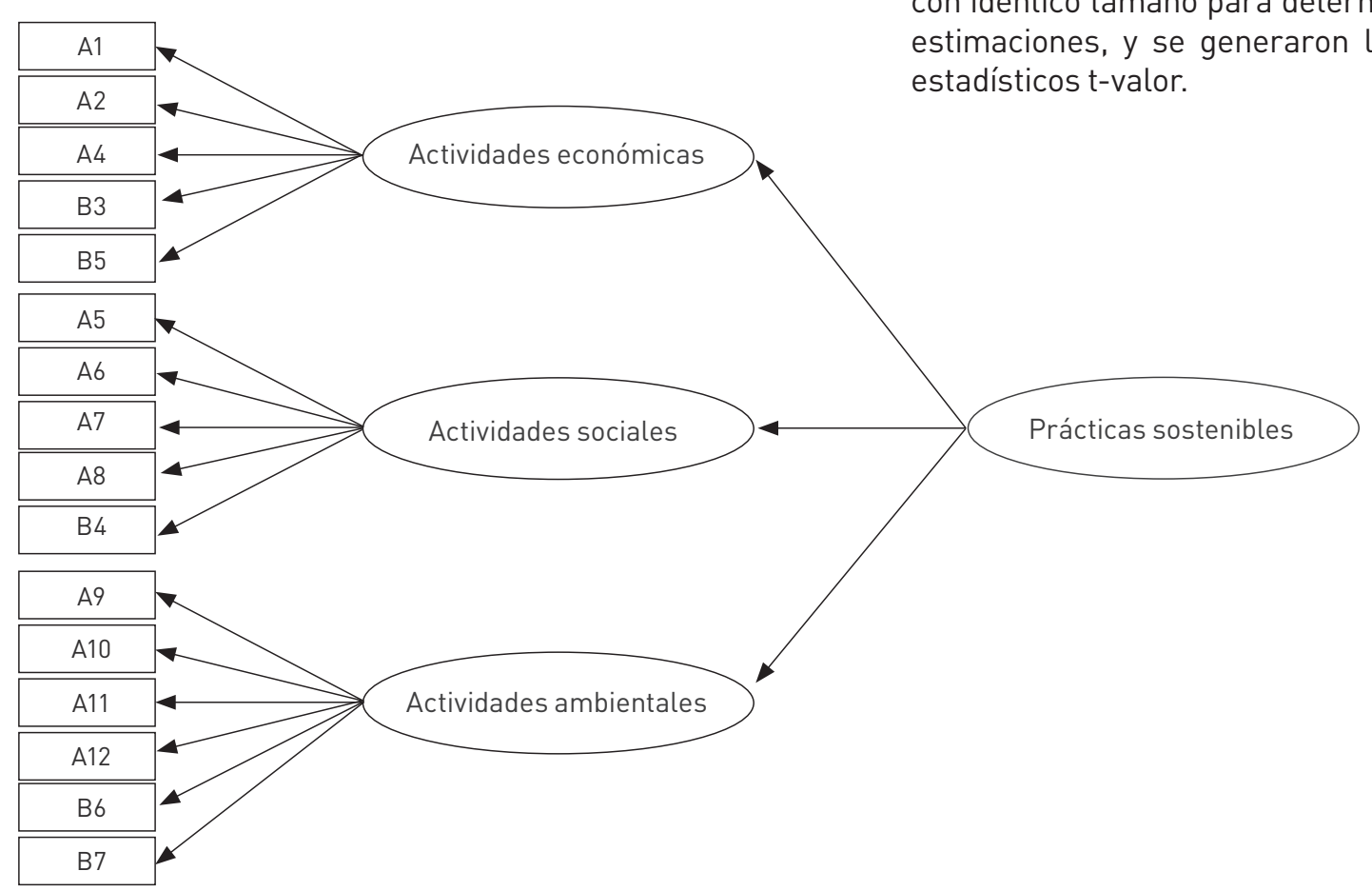

las tres dimensiones explican el $73,59 \%$ de la variabilidad total (KMO=0,883; prueba de esfericidad de Barlett: Chicuadrado=431,50 [gl=78], p-valor $<0,0001$ ).

La escala de calidad de servicio logístico queda compuesta por los cuatro ítems ya planteados, que explican el $68,88 \%$ de la variabilidad de la información. Por su parte, los cuatro ítems que componen la escala de satisfacción presentan cargas elevadas a un factor latente, lo que consigue explicar el $74,20 \%$ de la varianza. En ambos casos, la bondad del ajuste queda confirmada con los resultados obtenidos en la prueba de adecuación muestral KMO y en la prueba de Bartlett, ya que la medida KMO está muy próxima a la unidad ( $\mathrm{KMO}=0,678$ y 0,804, respectivamente) y el contraste de significatividad de Bartlett (Chi-cuadrado=96,13 $[\mathrm{gl}=6]$ y $\mathrm{Chi}$-cuadrado=112,06 $[\mathrm{gl}=6]$ ]) indica el rechazo de la hipótesis nula (Hair et al., 2008).

\subsection{Proceso de validación de las escalas de medida}

Una vez analizada la dimensionalidad de las medidas de manera exploratoria, se estimó el modelo de medida de segundo orden para plantear dos constructos reflectivos de segundo orden, sostenibilidad y valor logístico, que vienen configurados a partir de tres y tres constructos reflectivos de primer orden, respectivamente, junto con los factores unidimensionales (calidad y satisfacción) y las dos variables de control. La estimación se realizó mediante Partial Least Squares (PLS) (Ringle, Wende y Becker, 2015). Esta metodología permite suposiciones menos restrictivas que el enfoque basado en la covarianza, al comportar procedimientos no paramétricos. Siguiendo las recomendaciones de Henseler, Ringle y Sinkovics (2009), se empleó un bootstrapping con 5000 submuestras con idéntico tamaño para determinar la significación de las estimaciones, y se generaron los errores estándar y los estadísticos t-valor.

Figura 2. Dimensiones de la variable prácticas sostenibles Fuente: elaboración propia. 
Los resultados de la estimación, mostrados en la tabla 3 , permiten confirmar la fiabilidad de todas las escalas, ya que tanto los valores de la fiabilidad compuesta (FC) como del coeficiente alfa de Cronbach (AC) son superiores a los valores recomendados de 0,7 (Nunnally y Bernstein, 1994) o superiores a 0,8 (Carmines y Zeller, 1979). Para valorar la validez convergente se llevó a cabo el análisis de las cargas totales estandarizadas de los modelos de medida de primer y segundo orden. En el primer caso, las cargas asociadas a los ítems observados son superiores a 0,7 y estadísticamente significativas en el caso de las escalas unidimensionales. De manera similar, las dimensiones están correlacionadas de manera significativa, y además muestran cargas estadísticamente significativas sobre los segundos factores latentes - prácticas sostenibles y valor logístico-.

La varianza promedio extraída (AVE) es superior en todos los casos al valor crítico de 0,5 establecido en la literatura (Fornell y Larcker, 1981). Todo ello permite confirmar la fiabilidad y la validez convergente de las escalas de medida propuestas.

La validez discriminante, referida a que cada escala representa una dimensión separada, se confirma cuando la correlación entre dos factores latentes resulta inferior a la raíz cuadrada del AVE de cada uno de ellos (Fornell y Larcker, 1981). Tal y como se muestra en la tabla 4, los resultados indican que las escalas de medida están dotadas de validez discriminante.

\subsection{Estimación del modelo causal}

Confirmadas la multidimensionalidad de los constructos de prácticas sostenibles y de valor logístico, y la unidimensionalidad de los constructos de calidad de servicio y satisfacción, se propone el modelo objeto de análisis (figura 4). Como se indicó en el apartado 3.2, se completó la estimación añadiendo la antigüedad de la empresa y el tipo de negocio como variables de control.

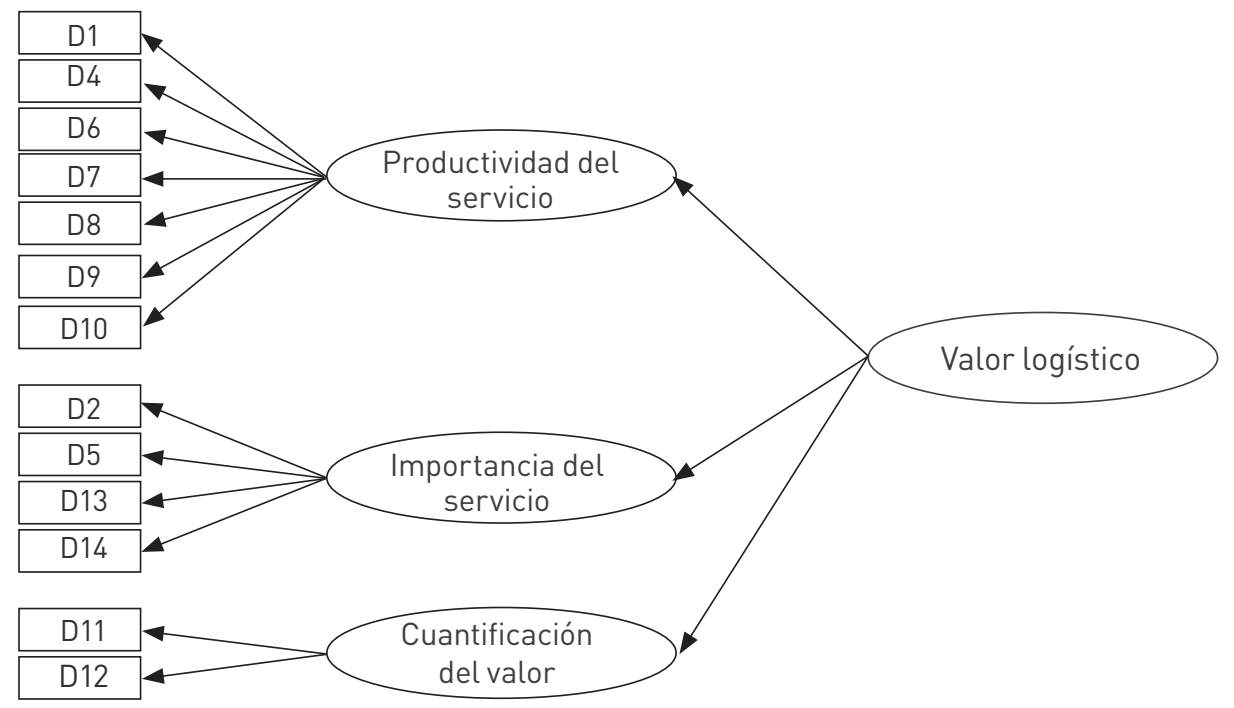

Figura 3. Dimensiones de la variable valor logístico Fuente: elaboración propia.

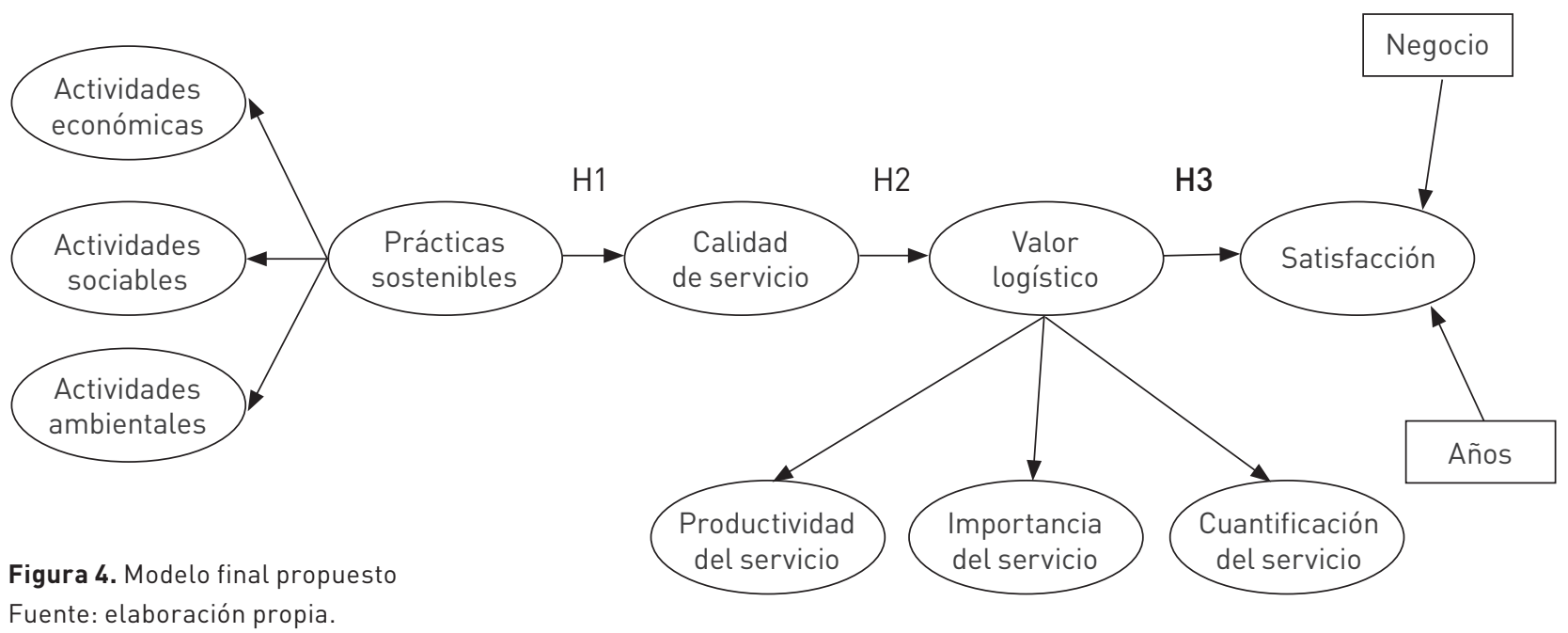

Fuente: elaboración propia. 
Tabla 3. Estimación del modelo de medida de segundo orden: fiabilidad, consistencia y validez convergente

\begin{tabular}{|c|c|c|c|c|c|c|}
\hline Constructo de primer orden & Indicador & Carga del factor & t-Valor & $A C$ & $\mathrm{FC}$ & AVE \\
\hline \multirow[t]{6}{*}{ ACTAMB <- Prácticas sostenibles } & A9 & $0,843^{* *}$ & 5,299 & 0,893 & 0,918 & 0,653 \\
\hline & $\mathrm{A} 10$ & $0,829 * *$ & 5,053 & & & \\
\hline & $\mathrm{A} 11$ & $0,690 * *$ & 2,837 & & & \\
\hline & $\mathrm{A} 12$ & $0,853^{* *}$ & 4,974 & & & \\
\hline & B6 & $0,831^{* *}$ & 4,021 & & & \\
\hline & B7 & $0,790 * *$ & 3,787 & & & \\
\hline \multirow[t]{5}{*}{ ACTECO <- Prácticas sostenibles } & $\mathrm{A} 1$ & $0,836^{* *}$ & 5,831 & 0,821 & 0,874 & 0,585 \\
\hline & $\mathrm{A} 2$ & $0,717^{* *}$ & 5,141 & & & \\
\hline & A4 & $0,820^{* *}$ & 6,494 & & & \\
\hline & B3 & $0,626^{* *}$ & 3,431 & & & \\
\hline & B5 & $0,803^{* *}$ & 4,581 & & & \\
\hline \multirow[t]{5}{*}{ ACTSOC <- Prácticas sostenibles } & A5 & $0,795^{* *}$ & 4,590 & 0,883 & 0,915 & 0,683 \\
\hline & A6 & $0,819 * *$ & 6,476 & & & \\
\hline & A7 & $0,823^{* *}$ & 5,095 & & & \\
\hline & A8 & $0,903^{* *}$ & 7,288 & & & \\
\hline & B4 & $0,786^{* *}$ & 5,064 & & & \\
\hline \multirow[t]{4}{*}{ Calidad } & $\mathrm{C} 1$ & $0,889 * *$ & 5,400 & 0,847 & 0,897 & 0,687 \\
\hline & $\mathrm{C} 2$ & $0,850^{* *}$ & 4,039 & & & \\
\hline & $\mathrm{C} 3$ & $0,842^{* *}$ & 8,730 & & & \\
\hline & $\mathrm{C} 4$ & $0,724^{* *}$ & 2,603 & & & \\
\hline \multirow[t]{4}{*}{ LSIMPORT <- Valor logístico } & $\mathrm{D} 2$ & 0,709 & 1,744 & 0,823 & 0,882 & 0,652 \\
\hline & D5 & $0,868^{* *}$ & 3,068 & & & \\
\hline & D13 & 0,798 & 1,618 & & & \\
\hline & D14 & $0,847^{* *}$ & 3,017 & & & \\
\hline \multirow[t]{2}{*}{ LSMEASURE <- Valor logístico } & D11 & $0,884^{* *}$ & 2,768 & 0,748 & 0,888 & 0,798 \\
\hline & D12 & $0,903^{* *}$ & 3,481 & & & \\
\hline \multirow[t]{7}{*}{ LSPRODUC <- Valor logístico } & D1 & $0,660 * *$ & 2,641 & 0,885 & 0,909 & 0,590 \\
\hline & D4 & 0,757 & 1,793 & & & \\
\hline & D6 & $0,675^{*}$ & 2,437 & & & \\
\hline & D7 & $0,829 *$ & 2,002 & & & \\
\hline & D8 & 0,840 & 1,658 & & & \\
\hline & D9 & 0,879 & 1,850 & & & \\
\hline & $\mathrm{D} 10$ & 0,707 & 1,634 & & & \\
\hline \multirow[t]{4}{*}{ Satisfacción } & E1_1 & $0,770^{* *}$ & 4,695 & 0,882 & 0,920 & 0,742 \\
\hline & E1_2 & $0,897^{* *}$ & 8,245 & & & \\
\hline & E1_3 & $0,873^{* *}$ & 5,188 & & & \\
\hline & E1_4 & $0,898^{* *}$ & 13,542 & & & \\
\hline \multicolumn{7}{|l|}{ Constructo de segundo orden } \\
\hline ACTAMB <- Prácticas sostenibles & ACTAMB & $0,898^{* *}$ & 11,384 & 0,839 & 0,853 & 0,671 \\
\hline ACTECO <- Prácticas sostenibles & ACTECO & $0,750^{* *}$ & 9,413 & & & \\
\hline ACTSOC <- Prácticas sostenibles & ACTSOC & $0,948^{* *}$ & 31,972 & & & \\
\hline LSIMPORT <- Valor logístico & LSIMPORT & $0,929 * *$ & 4,136 & 0,921 & 0,924 & 0,804 \\
\hline LSMEASURE <- Valor logístico & LSMEASURE & $0,906^{* *}$ & 2,748 & & & \\
\hline LSPRODUC <- Valor logístico & LSPRODUC & $0,951 * *$ & 8,169 & & & \\
\hline
\end{tabular}

Nota: $\mathrm{CA}=$ Alfa de Cronbach, FC=Fiabilidad compuesta, AVE= Varianza extraída promedio, ${ }^{* *} p<0,01,{ }^{*} p<0,05$. Fuente: elaboración propia. 
Tabla 4. Correlaciones entre constructos latentes (segundo orden)

\begin{tabular}{lllllll}
\hline & Años (control) & Calidad & Negocio (control) & Satisfacción & Sostenibilidad & Valor logístico \\
\hline Años (control) & --- & & & & & \\
Calidad & $-0,158$ & 0,828 & & & & \\
Negocio (control) & 0,063 & 0,123 & --- & & & \\
Satisfacción & $-0,137$ & 0,747 & 0,191 & 0,861 & 0,819 & \\
Sostenibilidad & $-0,127$ & 0,751 & $-0,062$ & 0,681 & 0,896 \\
Valor logístico & $-0,043$ & 0,755 & 0,256 & 0,721 & 0,657 & 0 \\
\hline
\end{tabular}

Fuente: elaboración propia.

A continuación, se estima el modelo de ecuaciones estructurales a través de PLS, y se comprueba el poder explicativo del modelo estructural a través de los coeficientes de determinación $\mathrm{R}^{2}$, que indica la cantidad de varianza de las variables endógenas explicada por los constructos y que toma valores entre 0 y 1 . Como se puede observar en la tabla 5, los valores superan el valor de 0,5 y los $Q^{2}$ son superiores a 0 , por lo que el modelo presenta un valor explicativo y predictivo adecuado, que permite, de este modo, evaluar la significatividad de las relaciones causales previamente establecidas.

Tabla 5. Estimaciones de las relaciones causales

\begin{tabular}{llll}
\hline Hipótesis & $\begin{array}{l}\text { B (Beta } \\
\text { estandarizado) }\end{array}$ & $\begin{array}{l}\text { Valor t } \\
\text { (Bootstrap) }\end{array}$ & Contraste \\
\hline $\begin{array}{l}\mathrm{H}_{1} \text { Sostenibilidad } \\
\rightarrow \text { Calidad }\end{array}$ & $0,751^{* *}$ & 6,914 & Aceptada \\
$\begin{array}{l}\mathrm{H}_{2} \begin{array}{l}\text { Calidad } \\
\rightarrow \text { Valor logístico }\end{array} \\
\mathrm{H}_{3} \begin{array}{l}\text { Valor Logístico } \\
\rightarrow \text { Satisfacción }\end{array}\end{array}$ & $0,646^{* *}$ & 4,030 & Aceptada \\
\hline
\end{tabular}

Nota: $R^{2}$ (Calidad) $=0,737 ; R^{2}$ (Satisfacción) $=0,531 ; R^{2}$ (Valor logístico) $=0,715 ; Q^{2}($ Calidad $)=0,464 ; Q^{2}($ Satisfacción $)=0,253 ; Q^{2}($ Valor logístico $)=$ 0,196 .

Fuente: elaboración propia.

Los resultados de la estimación del modelo permiten apoyar la existencia de una relación positiva entre las prácticas sostenibles y la calidad de servicio realizado por las compañías navieras; por lo tanto, se acepta la H1. Por otra parte, el estudio realizado confirma que la calidad de servicio tiene un efecto positivo sobre el valor logístico, tal como apunta el trabajo de Servera et al. (2008), que confirma la H2. En cuanto a la H3, que indica que el aumento del valor logístico incide directa y positivamente sobre la satisfacción del cliente, se acepta con base en las investigaciones realizadas por Eon-Seong y Dong-Wook (2010).

\section{Conclusiones}

Este trabajo se centra en analizar las prácticas de sostenibilidad desde el enfoque TBL leconómica, social y ambiental) y su influencia en la cadena de consecuentes formada por la calidad de servicio, el valor logístico y la satisfacción. Todo ello observado desde la óptica de la relación entre empresas (proveedor-cliente). La revisión de la literatura muestra tanto el interés como las carencias existentes en la investigación sobre este tema, en especial si el objeto de análisis es el transporte marítimo, articulado a un contexto geográfico como la República de Panamá, en donde este modo de transporte alcanza un protagonismo máximo. Este país tiene la marina mercante más grande del mundo, que alcanzó, en el 2018, el $16,6 \%$ de la flota mundial y registró un total de 6471 buques y 209,99 millones de toneladas. Por todo ello, este trabajo contribuye a cerrar esta brecha de información en la literatura, pues aclara cómo se construyen estas relaciones $\mathrm{B} 2 \mathrm{~B}$.

A partir de una muestra que incluye a expedidores, se ha planteado un modelo integrador para el análisis de los conceptos retenidos y sus relaciones. Dicho modelo confirma que las prácticas sostenibles impulsan la calidad del servicio y el valor logístico, al actuar de forma encadenada como variables antecedentes de la satisfacción. Confirma, además, que una buena relación entre proveedor y cliente (navieras y expedidores) conduce a una mayor satisfacción del cliente, y permite evidenciar las distintas contribuciones del trabajo.

En primer lugar, se ha confirmado que las prácticas sostenibles tienen efectos positivos y significativos sobre la calidad de servicio, así como su carácter multidimensional. En este sentido, se ha contribuido al avance del conocimiento en el estudio de la TBL en el ámbito del transporte marítimo, puesto que las prácticas sostenibles emergen como una única variable que contempla sus tres dimensiones base lacciones económicas, sociales y medioambientales). Se recalca que las empresas que deseen mejorar su productividad y ser competitivas deben preocuparse por aplicar prácticas de sostenibilidad desde un enfoque de TBL, $y$, en consecuencia, articular iniciativas apoyadas en sus tres pilares.

En segundo lugar, se concluye el efecto positivo y significativo de la calidad de servicio sobre el valor logístico. Esta relación sugiere que la calidad de servicio influye positivamente sobre el valor logístico, por lo que la calidad de servicio se convierte en una pieza clave en la generación de valor logístico. Del mismo modo, los resultados de este trabajo contribuyen a un mejor conocimiento relativo al valor logístico, por cuanto se constata que dicho valor se construye a partir de tres factores: productividad del servicio, importancia del servicio logístico y cuantificación del valor logístico. Estos factores corroboran los hallazgos previos de Gil-Saura, Servera-Francés y Fuentes-Blasco (2010) y enfatizan en el carácter multidimensional del valor en el transporte marítimo. Finalmente, el valor se muestra como un antecedente notable de la satisfacción del cliente, en cuanto expedidor. 
Con todo, los resultados obtenidos en esta investigación demuestran que la aplicación de prácticas sostenibles por parte de las compañías navieras representa desafíos y oportunidades únicas, por lo que se subraya su importancia como impulsoras de la calidad, del valor y de la satisfacción, pero, a su vez, también permite observar las dificultades en lo relativo a su comprensión, al detectarse una falta de conocimiento en torno a ellas por parte de las compañías navieras. En esta dirección, una recomendación para la gestión que emerge con claridad es la necesidad de comunicar mejor las acciones que se implementan desde la TBL, pues las percepciones de los clientes sobre las prácticas de sostenibilidad que aplican las navieras tienen efectos encadenados notables en términos de beneficios que redundan en última instancia en la satisfacción del expedidor.

En lo relativo a las limitaciones de este trabajo, el tamaño de la muestra no ha permitido realizar distinciones entre grupos, por ejemplo, entre exportadores, importadores o transitarios. Es clara la necesidad de ampliar el tamaño muestral y con ello considerar en futuros estudios la observación de diferencias entre estos grupos de empresas. Del mismo modo, podría ser recomendable analizar por separado cada actividad de sostenibilidad (económica, social y ambiental) y aplicar el análisis a este modelo. Finalmente, la investigación se ha limitado a un único ámbito geográfico, la industria del transporte marítimo de Panamá, lo que a su vez limita la validez externa de los resultados. Ampliar el estudio al incluir otros países permitiría adoptar un enfoque de carácter internacional y, con ello, tal vez abordar el análisis a partir de una dimensión cultural.

\section{Agradecimiento}

Este estudio ha sido realizado con el marco del Proyecto EC02016-76553-R del Ministerio de Educación y Ciencia del Gobierno de España. Agencia Estatal de Investigación.

\section{Conflicto de intereses}

Los autores declaran no tener ningún conflicto de intereses.

\section{Anexos}

Tabla A1. Escalas de Medición de las variables

\begin{tabular}{|c|c|c|c|c|c|}
\hline \multirow{2}{*}{\multicolumn{2}{|c|}{ Concepto / Ítem }} & \multicolumn{4}{|c|}{ Muestra total } \\
\hline & & $\begin{array}{l}\text { Valor } \\
\text { mínimo }\end{array}$ & $\begin{array}{l}\text { Valor } \\
\text { máximo }\end{array}$ & Media & $\begin{array}{l}\text { Desviación } \\
\text { típica }\end{array}$ \\
\hline \multicolumn{6}{|c|}{ Prácticas sostenibles } \\
\hline \multicolumn{6}{|c|}{ Actividades económicas } \\
\hline & Las actividades económicas de mi naviera contribuyen a el crecimiento económico de la sociedad. & 1 & 7 & 5,9 & 1,15 \\
\hline A2 & Mi naviera trata de aumentar el empleo de la comunidad local. & 1 & 7 & 5,64 & 1,31 \\
\hline A4 & $\begin{array}{l}\text { Mi naviera está aumentando sus ventas o su participación en el mercado mediante la introducción } \\
\text { de la innovación en la gestión. }\end{array}$ & 2 & 7 & 5,78 & 1,13 \\
\hline B3 & Mi naviera aplica altos estándares de divulgación, contabilidad e informes sociales y ambientales. & 2 & 7 & 5,16 & 1,27 \\
\hline B5 & Mi naviera cumple con las leyes y regulaciones fiscales en todos los países operativos. & 1 & 7 & 5,68 & 1,02 \\
\hline \multicolumn{6}{|c|}{ Actividades sociales } \\
\hline A5 & Mi naviera fomenta la cooperación con las comunidades regionales y las instituciones educativas. & 2 & 7 & 5,48 & 1,43 \\
\hline A6 & Mi naviera desarrolla su responsabilidad social corporativa en proporción a sus ventas. & 1 & 7 & 5,53 & 1,24 \\
\hline A7 & Mi naviera apoya la educación para su personal & 2 & 7 & 5,52 & 1,3 \\
\hline A8 & Mi naviera alienta a su personal a involucrarse en actividades de voluntariado en la comunidad. & 2 & 7 & 5,54 & 1,3 \\
\hline B4 & Mi naviera dona a organizaciones benéficas. & 2 & 7 & 5,4 & 1,14 \\
\hline \multicolumn{6}{|c|}{ Actividades medioambientales } \\
\hline A9 & $\begin{array}{l}\text { Mi naviera reduce las emisiones de } \mathrm{CO} 2 \text { mediante la reducción deliberada de la velocidad de sus } \\
\text { barcos. }\end{array}$ & 2 & 7 & 5,4 & 1,23 \\
\hline A10 & $\begin{array}{l}\text { Mi naviera administra adecuadamente el agua de lastre para proteger a los océanos de la } \\
\text { contaminación. }\end{array}$ & 1 & 7 & 5,46 & 1,36 \\
\hline A11 & Mi naviera cumple con los estándares internacionales establecidos por la OMI. & 1 & 7 & 5,94 & 1,13 \\
\hline A12 & Mi naviera presta mucha atención a la protección del medio ambiente. & 1 & 7 & 5,67 & 1,33 \\
\hline B6 & Mi naviera utiliza materiales y equipos respetuosos con el medio ambiente & 2 & 7 & 5,48 & 1,18 \\
\hline B7 & Mi naviera adopta diseños de construcción naval respetuosos con el medio ambiente. & 2 & 7 & 5,4 & 1,14 \\
\hline \multicolumn{6}{|c|}{ Valor logístico } \\
\hline \multicolumn{6}{|c|}{ Productividad del servicio } \\
\hline D1 & Estamos contentos con el nivel de servicio logístico que nos ofrece este proveedor. & 1 & 7 & 6 & 1,21 \\
\hline D4 & La mejora del servicio logístico es una alta prioridad en nuestra empresa. & 2 & 7 & 6,12 & 0,98 \\
\hline D6 & Comunicamos al proveedor que el servicio logístico ha excedido nuestras expectativas. & 2 & 7 & 5,9 & 1,09 \\
\hline D7 & Intentamos constantemente reducir el costo logístico global. & 1 & 7 & 6,06 & 1,08 \\
\hline D8 & $\begin{array}{l}\text { Alcanzar la productividad a través de la calidad de servicio logístico es crítico para nuestro } \\
\text { negocio. }\end{array}$ & 1 & 7 & 6,26 & 0,99 \\
\hline D9 & Estamos constantemente intentando incrementar el nivel de servicio logístico global. & 1 & 7 & 6 & 1,05 \\
\hline D10 & $\begin{array}{l}\text { La alta dirección de la empresa es consciente del impacto sobre las ventas de los cambios en el } \\
\text { nivel de servicio logístico. }\end{array}$ & 1 & 7 & 6,12 & 1,17 \\
\hline
\end{tabular}


Tabla A1. Escalas de Medición de las variables (continuación)

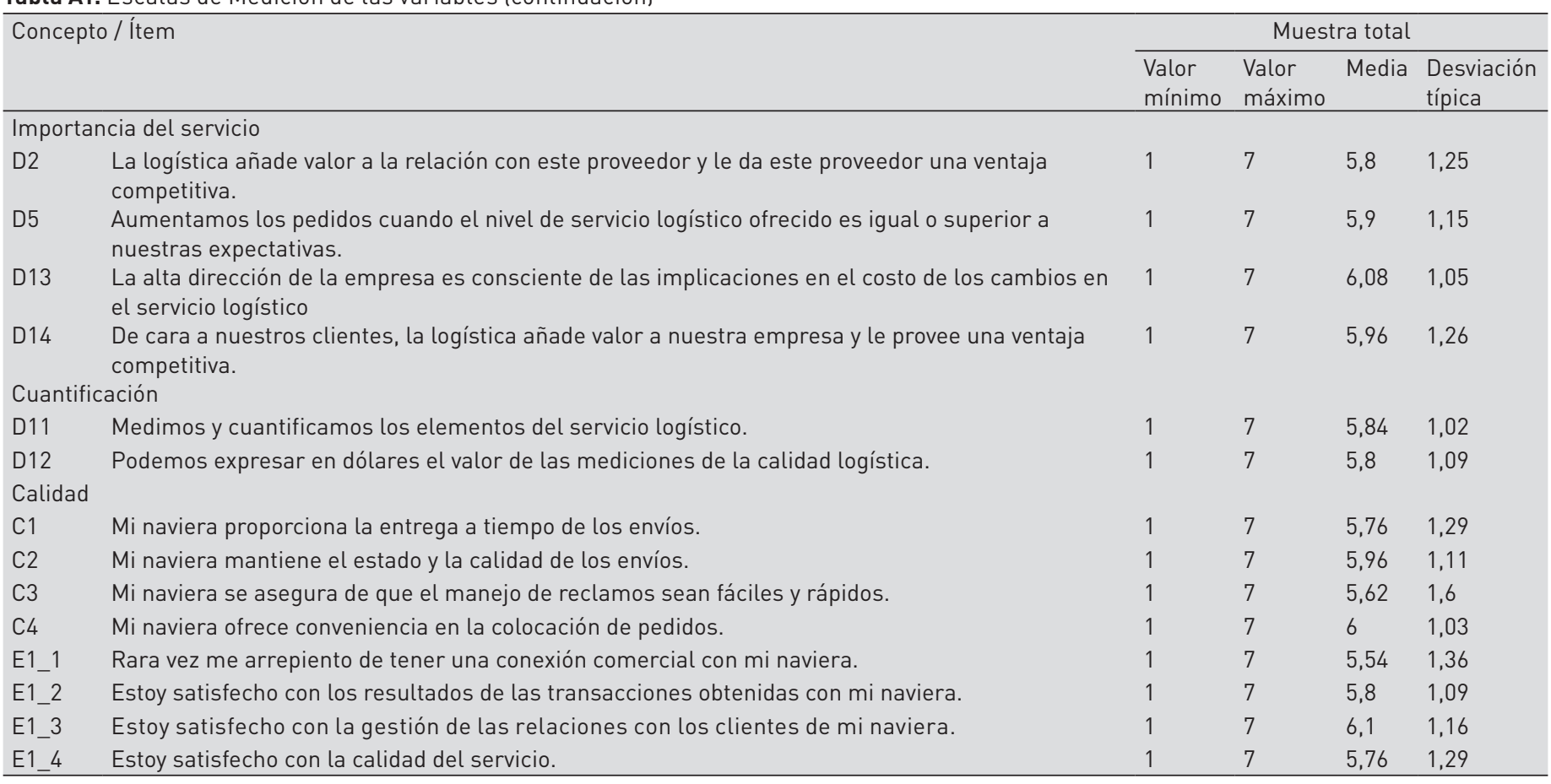

Fuente: elaboración propia.

\section{Bibliografía}

Ahi, P. y Searcy, C. (2015). Assessing sustainability in the supply chain: A triple bottom line approach. Applied Mathematical Modelling, 39l1011), 2882-2896. https://doi.org/10.1016/j.apm.2014.10.055

Asif, M., Searcy, C., Ambika, Z. y Ahmad, N. (2011). An integrated management systems approach to corporate sustainability. European Business Review, 23(4), 353-367. https://doi.org/10.1108/09555341111145744

Batista-Foguet, J. M. y Coenders-Gallart, G. (2000). Modelos de ecuaciones estructurales. Madrid: La Muralla.

Cabrera-Cánovas, A. (2011). Transporte internacional de mercancías. Madrid: ICEX

Carmines, E. G. y Zeller, R. A. (1979). Reliability and validity assessment. Thousand Oaks: Sage Publications.

Celik, M. (2009). Designing of integrated quality and safety management system (IQSMS) for shipping operations. Safety Science, 47(5), 569577. https://doi.org/10.1016/j.ssci.2008.07.002

Chen, K.-K., Chang, C.-T. y Lai, C.-S. (2009). Service quality gaps of business customers in the shipping industry. Transportation Research Part E: Logistics and Transportation Review, 45(1), 222-237. https://doi.org/10.1016/j.tre.2008.02.005

Cheng, T. C. E., Farahani, R. Z., Lai, K. H. y Sarkis, J. (2015). Sustainability in maritime supply chains: Challenges and opportunities for theory and practice. Transportation research Part E: Logistics and transportation review. https://doi.org/10.1016/j.tre.2015.03.007

Dyllick, T. y Hockerts, K. (2002). Beyond the business case for corporate sustainability. Business Strategy and the Environment, 11(2), 130-141. https://doi.org/10.1002/bse.323

Eon-Seong, L. y Dong-Wook, S. (2010). Knowledge management for maritime logistics value: Discussing conceptual issues. Maritime Policy and Management, 37(6), 563-583. https://doi.org/10.1080/03088839.2010.514959

Fornell, C. y Larcker, D. F. (1981). Structural equation models with unobservable variables and measurement error. Journal of Marketing Research, 18, 39-50. https://doi.org/10.2307/3151312

Frankel, E. G. (1993). Total quality management in liner shipping. Marine Policy, 17(1), 58-63. https://doi.org/10.1016/0308-597X(93)90006-0

Gil-Saura, I., Servera-Francés, D. y Fuentes-Blasco, M. (2010). Antecedents and consequences of logistics value: And empirical inves- tigation in the Spanish market. Industrial Marketing Management, 39(3), 493-506. https://doi.org/10.1016/j.indmarman.2008.11.007

Gil-Saura, I., Berenguer-Contri, G., Ruiz-Molina, M.-E. y Ospina-Pinzón, S. (2017). Methodologies for evaluating service quality: Evidence from freight services. International Journal of Transport Economics, 44(1). https://doi.org/10.19272/201706701005

Gil-Saura, I., Berenguer-Contri, G. y Ruiz-Molina, E. (2018). Satisfaction and loyalty in $\mathrm{B} 2 \mathrm{~B}$ relationships in the freight forwarding industry: Adding perceived value and service quality into equation. Transport, 33(5), 1184-1195. https://doi.org/10.3846/transport.2018.6648

Gounaris, S. (2005). Measuring service quality in B2B services: An evaluation of the SERVQUAL scale vis-a-vis the INDSERV scale. Journal of Services Marketing, 19(6), 421-435. https://doi.org/10.1108/08876040510620193

Hair, J. F. J., Anderson, R. E., Tatham, R. L. y Black, W. C. (2008). Análisis multivariante (5 $5^{\text {th }}$ ed.). Madrid: Prentice Hall.

Hammer, J. y Pivo, G. (2017). The Triple bottom line and sustainable economic development theory and practice. Economic Development Quarterly, 31(1), 25-36. https://doi.org/10.1177/0891242416674808

Helgesen, Ø. (2006). Are loyal customers profitable? Customer satisfaction, customer (action) loyalty and customer profitability at the individual level. Journal of Marketing Management, 22(3-4), 245-266. https://doi.org/10.1362/026725706776861226

Henseler, J., Ringle, C. y R. Sinkovics, R. (2009). The use of partial least squares path modeling in international marketing. Advances in International Marketing, 20(1), 277-319. https://doi.org/10.1108/S1474-7979(2009)0000020014

Hutchins, M. J. y Sutherland, J. W. (2008). An exploration of measures of social sustainability and their application to supply chain decisions. Journal of Cleaner Production, 16(15), 1688-1698. https://doi.org/10.1016/j.jclepro.2008.06.001

Kannan, V., Bose, S. K. y Kannan, N. G. (2011). An evaluation of ocean container carrier selection criteria: An Indian shipper's perspective. Management Research Review, 34(7), 754-772. https://doi.org/10.1108/01409171111146661

Kleindorfer, P. R., Singhal, K. y Van Wassenhove, L. N. (2005). Sustainable operations management. Production and Operations Management, 14(4), 482-492. https://doi.org/10.1111/j.1937-5956.2005.tb00235.x

Lam, J. S. L. y Lai, K. H. (2015). Developing environmental sustainability by ANP-QFD approach: The case of shipping operations. Journal of 
Cleaner Production, 105, 275-284.

https://doi.org/10.1016/j.jclepro.2014.09.070

Lindstad, H., Asbornslett, B.E. y Stromman, A.H. (2016). Opportunities for increased profit and reduced cost and emissions by service differentiation within container liner shipping. Maritime Policy \& Management. The flagship journal of international shipping and port research, 43(3), 280-294. https://doi.org/10.1080/03088839.2015.1038327

Lu, C.-S., Lin, C.-C. y Tu, C.-J. (2009). Corporate social responsibility and organisational performance in container shipping. International Journal of Logistics: Research and Applications, 12(2), 119-132. https://doi.org/https://doi.org/10.1080/13675560902749373

Lun, Y. H. V., Lai, K. H., Wong, C. W. Y. y Cheng, T. C. E. (2015). Greening and performance relativity: An application in the shipping industry. Computers and Operations Research, 54, 295-301. https://doi.org/10.1016/j.cor.2013.06.005

Lun, Y. H. V., Lai, K. H., Wong, C. W. Y. y Cheng, T. C. E. (2016). Green shipping management. Switzerland: Springer International Publishing.

Luttenberger, A. y Luttenberger, L. R. (2017). Sustainable procurement and environmental life-cycle costing in maritime transport. WMU Journal of Maritime Affairs, 16(2), 219-231.

https://doi.org/10.1007/s13437-016-0116-6

Mitchell, R. W., Wooliscroft, B. y Higham, J. (2010). Sustainable market orientation: A new approach to managing marketing strategy. Journal of Macromarketing, 30(2), 160-170. https://doi.org/10.1177/0276146710361928

Montabon, F., Sroufe, R. y Narasimhan, R. (2007). An examination of corporate reporting, environmental management practices and firm performance. Journal of operations management, 25(5), 9981014. https://doi.org/10.1016/j.jom.2006.10.003

Notteboom, T. E. (2006). The time factor in liner shipping services. Maritime Economics \& Logistics, 8(1), 19-39.

https://doi.org/10.1057/palgrave.mel.9100148

Novack, R. A., Langley Jr. C. J. y Rinehart, L. M. (1995). Creating logistics value: Themes for the future. Oak Brook, IL: Council of Logistics Management.

Nunnally, J. C. y Bernstein, I. H. (1994). Psychometric theory (3 ${ }^{\text {rd }}$ ed.). New York: McGraw-Hill.

Oliver, R. L. (1980). A cognitive model of the antecedents and consequences of satisfaction decisions. Journal of Marketing Research, 17(4), 460469. https://doi.org/10.1177/002224378001700405

Panayides, P.M. (2003). Competitive strategies and organizational performance in ship management. Maritime Policy \& Management, 30(2), 123-140. https://doi.org/10.1080/0308883032000084850

Parasuraman, A., Zeithaml, V. A. y Berry, L. L. (1985). A conceptual model of service quality and its implications for future research. Journal of Marketing, 49(4), 41-50. https://doi.org/10.1177/002224298504900403

Pawlik, T., Gaffron, P. y Drewes, P. A. (2012). Corporate social responsibility in maritime logistics. En D.-W. Song y P. M.Panayides (Eds.), Maritime Logistics (pp. 205-226). Emerald Group Publishing Limited. https://doi.org/10.1108/9781780523415-011

Psaraftis, H. N. (2016). Green Maritime Logistics: The Quest for Win-win Solutions. Transportation Research Procedia, 14, 133-142. https://doi.org/10.1016/j.trpro.2016.05.049

Ringle, C. M., Wende, S. y Becker, J.-M. (2015). SmartPLS 3. Bönningstedt: SmartPLS. Recuperado el 17 de julio de 2020, de: http://www.smartpls.com

Rutner, S. M. y Langley Jr. C. J. (2000). Logistics value: Definition, process and measurement. The International Journal of Logistics Management, 11(2), 73-82. https://doi.org/10.1108/09574090010806173

Sarkis, J., Helms, M. M. y Hervani, A. A. (2010). Reverse logistics and social sustainability. Corporate Social Responsibility and Environmental Management, 17(6), 337-354. https://doi.org/10.1002/csr.220

Schaltegger, S.y Burritt, R. (2014). Measuring and managing sustainability performance of supply chains: Review and sustainability supply chain management framework. Supply Chain Management, 19(3), 232-241. https://doi.org/10.1108/SCM-02-2014-0061

Schellinck, T. y Brooks, M. R. (2016). Does Superior service performance provided to shipping lines improve the perceived value of a port? International Journal of Shipping and Transport Logistics, 8(2), 175-193. https://doi.org/10.1504/IJSTL.2016.075009
Servera-Francés, D., Arteaga-Moreno, F. J. y Gil-Saura, I. (2011). Estimación de modelos causales con PLS: una aplicación al valor logístico. Estadística Española, 53(176), 93-126.

Servera-Francés, D., Gil-Saura, I. y Fuentes-Blasco, M. (2008). El valor logístico: una propuesta de modelo a partir de sus antecedentes y consecuencias. Revista Europea de Dirección y Economía de la Empresa, 17(1), 127-150.

Shin, Y. y Thai, V. V. (2015). The Impact of Corporate Social Responsibility on Customer Satisfaction, Relationship Maintenance and Loyalty in the Shipping Industry. Corporate Social Responsibility and Environmental Management, 22(6), 381-392. https://doi.org/10.1002/csr.1352

Shin, Y., Thai, V. V, Grewal, D. y Kim, Y. (2017). Do corporate sustainable management activities improve customer satisfaction, word of mouth intention and repurchase intention? Empirical evidence from the shipping industry. The International Journal of Logistics Management, 28(2), 555-570. https://doi.org/10.1108/IJLM-11-2015-0220

Srdoč, A., Bratko, I. y Sluga, A. (2007). Machine learning applied to quality management-A study in ship repair domain. Computers in Industry, 58(5), 464-473. https://doi.org/10.1016/j.compind.2006.09.013

Stindt, D. (2017). A generic planning approach for sustainable supply chain management - How to integrate concepts and methods to address the issues of sustainability? Journal of Cleaner Production, 153, 146-163. https://doi.org/10.1016/j.jclepro.2017.03.126

Sureshchandar, G. S., Rajendran, C. y Anantharaman, R. N. (2002). The relationship between service quality and customer satisfaction. A factor specific approach. Journal of Services Marketing, 16(4), 363-379.

Synnestvedt, S. y Schaltegger, T. (2002). The link between 'green' and economic success: environmental management as the crucial trigger between environmental and economic performance. Journal of Environmental Management, 65(3), 251-268. https://doi.org/10.1006/jema.2002.0555

Thai, V. (2008). Service quality in maritime transport: Conceptual model and empirical evidence. Asia Pacific Journal of Marketing and Logistics, 20(4), 493-518. https://doi.org/10.1108/13555850810909777

UNCTAD (2016). Informe sobre el transporte marítimo 2016. Recuperado el 17 de julio de 2020 de: https://unctad.org/es/PublicationsLibrary/rmt2016_es.pdf

UNCTAD (2018). Informe sobre el transporte marítimo 2018. Geneva. Recuperado el 17 de julio de 2020 de: https://unctad.org/es/PublicationsLibrary/rmt2018_es.pdf

Wan, J., Young, L., Kim, M., Ei, Y. y Kim, Y. M. (2016). Antecedents of adopting corporate environmental responsibility and green practices. Journalof Business Ethics, 148, 397-409 https://doi.org/10.1007/s10551-016-3024-y

Woodruff, R. B. (1997). Customer value: The next source for competitive advantage. Journal of the Academy of Marketing Science, 25(2), 139-153. https://doi.org/10.1007/bf02894350

Yuen, K. F. y Thai, V. V. (2015a). Service quality and customer satisfaction in liner shipping. International Journal of Quality and Service Sciences, 7(2/3), 170-183. https://doi.org/10.1108/IJQSS-02-2015-0024

Yuen, K. F. y Thai, V. V. (2015b). Service quality appraisal: a study of interactions. Total Quality Management and Business Excellence, 28178), 730-745. https://doi.org/10.1080/14783363.2015.1114881

Yuen, K. F. y Thai, V. V. (2016). The relationship between supply chain integration and operational performances: A study of priorities and synergies. Transportation Journal, 55(1), 31-50. https://doi.org/10.5325/transportationj.55.1.0031

Yuen, K. F. y Thai, V. V. (2017). Corporate social responsibility and service quality provision in shipping firms: financial synergies or tradeoffs? Maritime Policy and Management, 44(1), 131-146. https://doi.org/10.1080/03088839.2016.1237782

Yuen, K. F., Wang, X., Wong, Y. D. y Zhou, Q. (2017). Antecedents and outcomes of sustainable shipping practices: The integration of stakeholder and behavioural theories. Transportation Research Part E: Logistics and Transportation Review, 108, 18-35. https://doi.org/10.1016/j.tre.2017.10.002 